\title{
Avaliação do trauma cranioencefálico em um hospital de urgência e emergência do Estado de Sergipe
}

Evaluation of traumatic brain injury in a hospital of urgency and emergency in the State of Sergipe

Evaluación de traumatismo craneoencefálico en un hospital de urgencia y emergencia del Estado de Sergipe

Recebido: 31/12/2020 | Revisado: 02/01/2021 | Aceito: 07/01/2021 | Publicado: 08/01/2021

Edna Santos Dias

ORCID: https://orcid.org/0000-0001-5888-0889

Universidade Tiradentes, Brasil

E-mail: ednasdc.enf@gmail.com

Carla Viviane Freitas de Jesus

ORCID: https://orcid.org/0000-0002-7775-6610 Universidade Tiradentes, Brasil

E-mail: carlavfj@gmail.com

Yasmim Anayr Costa Ferrari

ORCID: https://orcid.org/0000-0003-1766-341X Universidade Federal de Sergipe, Brasil

E-mail: yasmimanayr@hotmail.com

Elder Cardoso Ferreira

ORCID: https://orcid.org/0000-0002-8240-4329

Universidade Tiradentes, Brasil

E-mail: eldercf@icloud.com

Rute Nascimento da Silva

ORCID: https://orcid.org/0000-0002-2719-1623

Universidade Tiradentes, Brasil

E-mail: silva_rute@hotmail.com

Ana Caroline Gusmão de Matos

ORCID: https://orcid.org/0000-0002-4451-0243

Universidade Tiradentes, Brasil

E-mail: anagusmaao@gmail.com

Denison Pereira da Silva

ORCID: https://orcid.org/0000-0001-7038-6496 Universidade Tiradentes, Brasil

E-mail: denisonbm@yahoo.com.br

Cristiane de Alencar Domingues

ORCID: https://orcid.org/0000-0002-2163-0672

Universidade de São Paulo, Brasil

E-mail: crismingues@gmail.com

Lilia de Souza Nogueira

ORCID: https://orcid.org/0000-0001-5387-3807

Universidade de São Paulo, Brasil

E-mail: lilianogueira@usp.br

Sonia Oliveira Lima

ORCID: https://orcid.org/0000-0002-3257-2412

Universidade Tiradentes, Brasil

E-mail: sonialima.cirurgia@gmail.com

\section{Resumo}

Objetivo: Avaliar o perfil das vítimas trauma cranioencefálico (TCE) em um hospital de urgência e emergência do estado de Sergipe. Métodos: Trata-se de um estudo longitudinal, observacional do tipo prospectivo, com abordagem quantitativa das vítimas de TCE que deram entrada no eixo crítico de um hospital do estado de Sergipe, na cidade de Aracaju. Foi utilizado um instrumento de elaboração própria, tendo como base o instrumento de Domingues (2013), que contém questões objetivas com dados sociodemográficos e fisiológicos, referente à topografia e informações clínicas do estudo. Resultados: Da amostra de 192 pacientes foi vista uma média de idade de 36,6 anos, com 87,5\% do sexo masculino e $12,5 \%$ do sexo feminino, onde $74 \%$ sofreu acidente de transporte, a minoria de mecanismo contuso. $(96,4 \%)$ das vítimas recebeu atendimento pré-hospitalar, com $(59,9 \%)$ tendo alta como desfecho enquanto $34,4 \%$ foram à óbito, os pacientes tiveram um tempo mediano de internação de 11 dias, com $(54,2 \%)$ deles sem a necessidade de internamento em UTI, a minoria deles teve um desfecho sem sequelas. Conclusão: O TCE ainda tem como principal causa o acidente de transporte, com uma prevalência de trauma contuso, onde o perfil geral das vítimas foram predominantemente pacientes do sexo masculino, adultos jovens na faixa etária entre 18 e 29 anos. 
Mesmo com a maioria dos pacientes recebendo atendimento pré-hospitalar, as taxas de óbito, embora mais baixas, ainda estão bem próximas das taxas de alta hospitalar.

Palavras-chave: Traumatismo cranioencefálico; Epidemiologia; Traumatismos craniocerebrais.

\begin{abstract}
Objective: To evaluate the profile of victims traumatic brain injury (TBI) in an emergency hospital in the state of Sergipe. Methods: This is a longitudinal, observational, prospective study, with a quantitative approach to TBI victims who were admitted to the critical axis of a hospital in the state of Sergipe, in the city of Aracaju. A self-made instrument was used, based on the Domingues instrument (2013), which contains objective questions with sociodemographic and physiological data, referring to the topography and clinical information of the study. Results: From the sample of 192 patients, an average age of 36.6 years was seen, with $87.5 \%$ male and $12.5 \%$ female, where $74 \%$ suffered a transport accident, the minority of a blunt mechanism. (96.4\%) of the victims received pre-hospital care, with (59.9\%) being discharged as an outcome while $34.4 \%$ died, patients had a median hospital stay of 11 days, with $(54,2 \%)$ of them without the need for ICU admission, the minority of them had an outcome without sequelae. Conclusion: TBI is still the main cause of transport accidents, with a prevalence of blunt trauma, where the general profile of the victims was predominantly male patients, young adults aged between 18 and 29 years. Even with the majority of patients receiving prehospital care, death rates, although lower, are still very close to discharge rates.
\end{abstract}

Keywords: Traumatic brain injury; Epidemiology; Craniocerebral trauma.

\begin{abstract}
Resumen
Objetivo: Evaluar el perfil de víctimas de traumatismo craneoencefálico (TCE) en un hospital de urgencias del estado de Sergipe. Métodos: se trata de un estudio longitudinal, observacional, prospectivo, con abordaje cuantitativo a víctimas de TCE que ingresaron al eje crítico de un hospital del estado de Sergipe, en la ciudad de Aracaju. Se utilizó un instrumento de elaboración propia, basado en el instrumento Domingues (2013), que contiene preguntas objetivas con datos sociodemográficos y fisiológicos, referentes a la topografía e información clínica del estudio. Resultados: De la muestra de 192 pacientes se observó una edad promedio de 36,6 años, con 87,5\% hombres y 12,5\% mujeres, donde $74 \%$ sufrió un accidente de transporte, la minoría de un mecanismo contundente. (96,4\%) de las víctimas recibieron atención prehospitalaria, siendo $(59,9 \%)$ el alta como resultado, mientras que el 34,4\% falleció, los pacientes tuvieron una estancia hospitalaria media de 11 días, con $(54,2 \%)$ de ellos sin necesidad de ingreso en UCI, la minoría de ellos tuvo un resultado sin secuelas. Conclusión: El TCE sigue siendo la principal causa de accidentes de transporte, con prevalencia de traumatismo cerrado, donde el perfil general de las víctimas fue predominantemente masculino, adultos jóvenes de entre 18 y 29 años. Incluso con la mayoría de los pacientes que reciben atención prehospitalaria, las tasas de mortalidad, aunque más bajas, siguen estando muy cerca de las tasas de alta.
\end{abstract}

Palabras clave: Traumatismo craneoencefálico; Epidemiología; Traumatismos craneocerebrales.

\title{
1. Introdução
}

O trauma é a terceira causa de morte da população mundial como um todo, sendo superado apenas pela doença cardiovascular e as neoplasias (Quevedo, 2009; Blackwell, Lucas \& Clarke, 2014). As Lesões decorrentes do trauma mecânico variam desde pequenas feridas isoladas até lesões de alta complexidade, implicando em um risco multissistêmico. O paciente gravemente ferido, deve ser classificado como politraumatizado, havendo a necessidade de uma abordagem sistemática para a gestão, e assim um melhor resultado como desfecho (Raja \& Zane, 2018).

Dentre as lesões decorrentes do trauma, o traumatismo cranioencefálico (TCE) é destacado como um dos de maior gravidade, de principais causas de óbito, de incapacidade física e mental entre adultos jovens em todo o mundo. O TCE é superado apenas pelo acidente vascular cerebral como doença neurológica com maior impacto na qualidade de vida (Pereira, Duarte \& Santos, 2006; Raja \& Zane, 2018). O TCE pode ser classificado, quanto ao mecanismo em fechado ou penetrante, quanto a gravidade, em leve, moderado ou grave, e em relação à morfologia, em: extracranianas, cranianas e intracranianas (Sousa, 2006).

Para a melhoria na qualidade dos sistemas de atendimento a vítima traumatizada são utilizados programas de melhoria de qualidade, cujas bases são os registros de trauma, utilizando os índices de gravidade. Os índices de trauma são sistemas que permitem mensurar os níveis de lesões anatômicas e suas repercussões fisiológicas. Existe uma grande diversidade, e entre eles temos o Trauma and Injury Severity Score (TRISS) que é verificado através do Revisited Trauma Score (RTS) (Estumano et al., 2015). 
O RTS é um índice fisiológico, analisado no momento da admissão hospitalar da vítima através do escore da Escala de Coma de Glasgow (ECG), valor da pressão arterial sistólica (PAS) e valor da frequência respiratória (FR). O TRISS-like utiliza as variáveis MRM (Melhor resposta motora), PAS (Pressão artéria sistólica), Injury Severity Score (ISS), idade e mecanismo de trauma, é verificado em pacientes em uso de ventilação mecânica, uma vez que estes não eram considerados no TRISS (Domingues et al., 2013).

Os pacientes acometidos de TCE, apresentam tempo de tratamento e reabilitação mais prolongados e necessitam de maior tempo de internação, além das sequelas posteriores que os impossibilitam de executar suas funções, tendo impacto negativo na saúde e na economia do País. É importante, portanto, um estudo de caráter epidemiológico para avaliar e caracterizar como o TCE se apresenta no estado de Sergipe, além de relacionar ao uso dos métodos TRISS, e o TRISS-like, devido à escassez de dados relacionados ao uso desses métodos. Objetivou-se Avaliar o perfil das vítimas trauma cranioencefálico (TCE) em um hospital de urgência e emergência do estado de Sergipe (HUSE) no período de agosto de 2018 a julho de 2019.

\section{Metodologia}

Trata-se de um estudo longitudinal, observacional do tipo prospectivo, com abordagem quantitativa das vítimas de Trauma Crânio Encefálico (TCE) que deram entrada no eixo crítico de um hospital do estado de Sergipe no período de Agosto de 2018 a Julho de 2019.

A população foi composta por pacientes que sofreram trauma mecânico contuso e/ou penetrante admitidos com TCE e com idade igual ou superior de 18 anos atendidos no eixo crítico do pronto socorro do HUSE, e que precisaram de internamento hospitalar ou foram submetidos a tratamento cirúrgico e que o paciente ou o responsável aceitaram assinar o termo de consentimento livre esclarecido autorizando sua inclusão na pesquisa. Foram excluídos pacientes que apresentaram outros tipos de traumas decorrentes exclusivamente por queimaduras, afogamentos e aqueles cujos dados foram incompletos prejudicando a interpretação da pesquisa.

Para o cálculo da estimativa mínima necessária da amostra, do presente estudo, foi utilizada a fórmula de Pocock (1983). Considerou-se a contagem de paciente vítimas de TCE e que deram entrada no eixo crítico do pronto socorro do HUSE, no ano de 2018. Segundo dados da gestão do Hospital, neste ano, foram atendidas uma média 370 vítimas, com idade igual ou maior de 18 anos, que sofreram TCE e necessitaram de hospitalização com ou sem intervenção cirúrgica. Desta forma, a amostra mínima calculada para este estudo foi de 188. No período estudado foram incluídos 192 pacientes admitidos no eixo crítico.

Para a coleta dos dados das vítimas atendidas no eixo crítico do HUSE foi aplicado um instrumento de elaboração própria, utilizando como base o instrumento de Domingues (2013), que contém questões objetivas com dados sociodemográficos e fisiológicos, referente à topografia e informações clínicas do estudo.

Diante das alterações anatômicas e fisiológicas em decorrência do trauma as vítimas foram avaliadas através dos métodos TRISS (Trauma Injury Severity Score) e TRISS-like, verificou se o paciente permaneceu no setor critico (ala vermelha) ou se foi encaminhado para Unidade de Terapia Intensiva (UTI); Centro cirúrgico (C.C); exames por imagem. A vítima foi acompanhada até seu desfecho final: alta, óbito, evasão ou transferência.

Os índices de gravidade mais utilizados no trauma são obtidos a partir de informações da vítima no momento da admissão hospitalar, utilizando medidas anatômicas e fisiológicas como o TRISS. Para o cálculo do TRISS são necessários os índices de ISS (Injury Severity Score) e RTS (Revised Trauma Score), considerando ainda a idade da vítima e o tipo de trauma se é contuso ou penetrante (Estumano et al., 2015).

O RTS é um índice fisiológico, analisado no momento da admissão hospitalar da vítima através do escore da Escala 
de Coma de Glasgow (ECG), valor da pressão arterial sistólica (PAS) e valor da frequência respiratória (FR). O cálculo da probabilidade de sobrevida é realizado pela soma dos produtos resultantes dos valores atribuídos aos três componentes, multiplicados por respectivos pesos. Quanto maior o valor, melhor será o prognóstico, as variáveis variam de 0 a 4 , sendo 4 seu melhor prognóstico. E os valores da probabilidade de sobrevida pelo RTS variam de 0 a 8 , sendo 8 seu melhor prognóstico e os valores das variáveis devem ser analisados e somados conforme a fórmula: RTS $=0,9368 \times \mathrm{ECG}+0,7326 \times \mathrm{PAS}+0,2908 \times$ FR (Champion et al., 1990).

O ISS é um índice anatômico que avalia a gravidade do trauma, cujo cálculo é baseado na Abbreviated Injury Scale (AIS). Para caracterizar os valores da AIS, utiliza-se um método anatômico que avalia os ferimentos e que determina, AIS=1 são definidas como "leves", as AIS=2, "moderadas", as AIS=3, "graves", as AIS=4, "muito graves", as AIS=5, "críticas" e as AIS=6, letais. Em uma escala de um (lesão leve) e seis (lesão grave, normalmente fatal), a gravidade de cada lesão traumática segundo regiões do corpo, determinado pelo exame físico, exames radiológicos e cirurgias. O ISS considera as lesões existentes em seis regiões do corpo, sendo: 1-cabeça e pescoço; 2-face; 3-tórax; 4- abdômen ou conteúdo pélvico; 5 extremidades ou cintura pélvica; 6-superfície externa) e a soma do quadrado dos maiores valores da AIS de três regiões distintas define o escore final do ISS (Parreira et al., 2014). O valor AIS refere-se ao escore da gravidade de cada lesão, descrito no dicionário AIS, essa gravidade varia de 1 a 6. No presente estudo, para codificar as lesões foi utilizado o manual AIS versão (2015).

Para saber a Probabilidade de sobrevida identificada pelo TRISS é calculada através da fórmula: Ps $=1 /(1+e-b)$. Seus valores são: valor de 'e'= (2,718282) onde "e" representa a base do logaritmo neperiano; o valor de 'b' é originado da equação que considera valores do RTS, ISS, idade e distintos coeficientes segundo o tipo de trauma (contuso ou penetrante) e é calculado através da equação: $b=b 0$ + b1 (RTS) + b2 (ISS) + b3 (idade). Com relação à idade, o TRISS considera, se menor ou igual a 54 anos (idade $=$ zero) ou se maior de 54 anos (idade = 1) (Domingues, 2013; Champion et al., 1990).

O TRISS-like é uma adequação do modelo TRISS que se utiliza as variáveis MRM (Melhor resposta motora), PAS (Pressão artéria sistólica), ISS, idade e mecanismo de trauma, ambos obtidos a admissão hospitalar. Nessa adequação inclui-se pacientes em uso de Ventilação mecânica, uma vez que estes não eram considerados no TRISS, no qual o modelo inviabilizava a obtenção de valores fidedignos da FR e da ECG justificado pela utilização de Intubações endotraqueais, sedações e bloqueadores neuromusculares.

O TRISS-like é calculado através da equação: $\mathrm{b}=\mathrm{b} 0+\mathrm{b} 1(\mathrm{MRM})+\mathrm{b} 2(\mathrm{PAS})+\mathrm{b} 3$ (ISS) +b4(Idade) (Domingues, 2013). Os pesos b0, b1, b2, b3 e b4 foram derivados do modelo de regressão logística, para trauma contuso e penetrante.

Os dados foram alimentados em uma planilha do Statistical Package for the Social Sciences (SPSS) verão 21.0. Para a análise descritiva das variáveis foram utilizados porcentagem, frequência, média e desvio padrão, expostas em tabelas. O cálculo dos valores obtidos dos índices: TRISS foi feito a partir dos resultados adquiridos pela calculadora online do site trauma.org e o TRISS-like foi obtido por meio de regressão logística múltipla entre desfecho e MRM, ISS, pressão arterial sistólica e faixa etária. Foram considerados significativos quando $p<0,05$. Nas variáveis independentes foram utilizados o teste $\mathrm{x}^{2}$. Nas dependentes foram aplicados os testes de Mann Withney, teste de Fisher, teste Qui-quadrado de Pearson, teste de Qui-quadrado de Pearson com simulações de Monte-Carlo nas variáveis continuas não normais e Razão de Chances ajustadas.

A pesquisa foi aprovada pelo Comitê de Ética em Pesquisa da Universidade Tiradentes sobre o protocolo número 2.837.862.

\section{Resultados}

No período de agosto de 2018 a Julho de 2019, foram admitidos no setor crítico do HUSE 192 pacientes vítimas de Trauma Crânio Encefálico (TCE). 
A Tabela 1 mostra que existiu uma prevalência de pacientes do sexo masculino (87,5\%), com idade entre 18-29 anos $(39,1 \%)$, apresentando uma média de 36,6 anos $(\mathrm{DP}=15,2)$ anos. Destas vítimas, $(74 \%)$ sofreram acidente de transporte, sendo o mecanismo de trauma contuso o mais predominante $(89,1 \%)$. O atendimento pré-hospitalar foi recebido por $(96,4 \%)$ das vítimas.

Tabela 1 - Distribuição dos pacientes vítimas de TCE admitidos no setor crítico do HUSE, no período de Agosto de 2018 a Julho de 2019, segundo características do trauma. Aracaju/SE, Brasil (N=192).

\begin{tabular}{lr}
\hline Faixa etária & $\mathbf{N}(\%)$ \\
$18-29$ & $75(39,1)$ \\
$30-44$ & $67(34,9)$ \\
$45-59$ & $36(18,8)$ \\
$>60$ & $14(7,3)$ \\
Sexo & \\
Masculino & $168(87,5)$ \\
Feminino & $24(12,5)$ \\
Mecanismo de trauma & $171(89,1)$ \\
Contuso & $21(10,9)$ \\
Penetrante & $6(3,1)$ \\
Causa externa & $16(8,3)$ \\
FAB & $142(74,0)$ \\
FAF & $14(7,3)$ \\
Acidente de transporte & $14(7,3)$ \\
Agressão & $185(96,4)$ \\
Queda & $7(3,6)$ \\
Atendimento pré-hospitalar & \\
Sim & \\
Não &
\end{tabular}

Fonte: Autores.

A Tabela 2 mostra que, além do TCE, as vítimas sofreram também outras alterações anatômicas e fisiológicas, as lesões encontradas nos pacientes (45,8\%) foram em face, seguidas das lesões em extremidades ou cintura pélvica (46,9\%), e logo depois, em tórax $(22,4 \%)$. Foi observado também que o ISS médio dos pacientes foi de 13,4 (DP=9,7), a pontuação média na ECG foi de 14,6 (12,1), havendo uma prevalência no escore entre 13 -15 (65,3\%). A pressão arterial sistólica teve uma média de 122,5 mmHg (DP=26,7 mmHg), onde na maioria se encontrava acima de $89 \mathrm{mmHg}(94,3 \%)$. Foram submetidos a procedimento cirúrgico antes de $24 \mathrm{~h} 81(42,4 \%)$ das vítimas, onde 41 (50,6\%) destes procedimentos foram realizados em região de cabeça, 27(33,3\%) para reparação, fixação ou reposicionamento, além de 13 (16\%) dos procedimentos realizados em região de tórax. Os pacientes que tiveram complicações representaram um total de $(16,4 \%)$ sendo em sua maioria pneumonia $(32,3 \%)$, seguida de paralisia $(22,6 \%)$ e $(19,6)$ com lesões por pressão (LPP). Com um tempo médio de internação foi de 11 dias e a probabilidade de sobrevivência média foi de 39,3\% ( $\mathrm{DP}=45,8 \%)$, houve uma prevalência $(59,9 \%)$ de pacientes que tiveram alta, enquanto $(34,4 \%)$ foram à óbito, predominando pacientes que não foram internados em UTI $(54,2 \%)(p<0,017)$. 
Tabela 2 - Distribuição dos pacientes vítimas de TCE admitidos no setor crítico do HUSE, no período de Agosto de 2018 a Julho de 2019, segundo características das regiões corpóreas acometida, evento traumático, consequências e desfecho. Aracaju/SE, Brasil. ( $\mathrm{N}=192)$.

\begin{tabular}{|c|c|}
\hline & $\mathbf{N}(\%)$ \\
\hline \multicolumn{2}{|l|}{ AIS } \\
\hline Face & $88(45,8)$ \\
\hline Tórax & $43(22,4)$ \\
\hline Abdomen ou Conteúdo Pélvico & $18(9,4)$ \\
\hline Extremidades ou Cintura Pélvica & $90(46,9)$ \\
\hline Superfície Externa & $8(4,2)$ \\
\hline \multicolumn{2}{|l|}{ COD.PAS } \\
\hline $50-75 \mathrm{mmHg}$ & $7(3,6)$ \\
\hline $76-89 \mathrm{mmHg}$ & $4(2,1)$ \\
\hline$>89 \mathrm{mmHg}$ & $181(94,3)$ \\
\hline \multicolumn{2}{|l|}{ Fez procedimento cirúrgico em $<24$ horas } \\
\hline Sim & $81(42,4)$ \\
\hline Não & $110(57,6)$ \\
\hline \multicolumn{2}{|l|}{ Qual procedimento } \\
\hline Drenagem & $4(4,9)$ \\
\hline Cabeça & $41(50,6)$ \\
\hline Tórax & $13(16,0)$ \\
\hline Abdômen & $6(7,4)$ \\
\hline Amputações & $1(1,2)$ \\
\hline Reparação/Fixação/Reposicionamento & $27(33,3)$ \\
\hline \multicolumn{2}{|l|}{ Sequelas } \\
\hline Não & $158(83,6)$ \\
\hline Sim & $31(16,4)$ \\
\hline Pneumonia & $10(32,3)$ \\
\hline Retirada cirúrgica & $2(6,5)$ \\
\hline Paralisia & $7(22,6)$ \\
\hline Morte Encefálica & $3(9,7)$ \\
\hline Sepse & $1(3,2)$ \\
\hline Lesão por pressão & $6(19,4)$ \\
\hline Não especificada & $4(12,9)$ \\
\hline \multicolumn{2}{|l|}{ Internação em UTI } \\
\hline $\operatorname{Sim}$ & $88(45,8)$ \\
\hline Não & $104(54,2)$ \\
\hline \multicolumn{2}{|l|}{ Desfecho } \\
\hline Óbito & $66(34,4)$ \\
\hline Alta & $115(59,9)$ \\
\hline Transferência & $8(4,2)$ \\
\hline Evasão & $3(1,6)$ \\
\hline Probabilidade de sobrevida, média (DP) & $39,3(45,8)$ \\
\hline
\end{tabular}

Fonte: Autores.

A Tabela 3 apresenta os resultados comparativos entre os pacientes vítimas de TCE dos grupos avaliados pelos métodos TRISS-like $(\mathrm{n}=120)$ e TRISS $(\mathrm{n}=72)$, sem diferença significativa entre a idade, faixa etária, os sexos, os mecanismos de trauma, causas externas e atendimento pré-hospitalar. 
Tabela 3 - Dados comparativos entre as vítimas de TCE do grupo TRISS-like e do grupo TRISS admitidos no setor crítico do HUSE, no período de Agosto de 2018 a Julho de 2019, segundo características do trauma. Aracaju/SE, Brasil. (N=192).

\begin{tabular}{|c|c|c|c|}
\hline & \multicolumn{2}{|c|}{ Tipo } & \multirow{3}{*}{ p-valor } \\
\hline & & TRISS $(n=72)$ & \\
\hline \multicolumn{3}{|c|}{$\begin{array}{c}\text { TRISS-like } \\
(n=120)\end{array}$} & \\
\hline Idade, média $(D P)$ & $37,1(15,1)$ & $35,6(15,4)$ & $0,335^{\mathrm{w}}$ \\
\hline \multicolumn{4}{|l|}{ Faixa etária, $n(\%)$} \\
\hline $18-29$ & $41(34,2)$ & $34(47,2)$ & \multirow{4}{*}{$0,155^{\mathrm{QM}}$} \\
\hline $30-44$ & $47(39,2)$ & $20(27,8)$ & \\
\hline $45-59$ & $25(20,8)$ & $11(15,3)$ & \\
\hline$>60$ & $7(5,8)$ & $7(9,7)$ & \\
\hline \multicolumn{4}{|l|}{ SEXO, $n(\%)$} \\
\hline Masculino & $106(88,3)$ & $62(86,1)$ & \multirow[t]{2}{*}{$0,658^{\mathrm{F}}$} \\
\hline Feminino & $14(11,7)$ & $10(13,9)$ & \\
\hline \multicolumn{4}{|c|}{ Mecanismo de trauma, $n(\%)$} \\
\hline Contuso & $108(90,0)$ & $63(87,5)$ & \multirow[b]{2}{*}{$0,637^{\mathrm{F}}$} \\
\hline Penetrante & $12(10,0)$ & $9(12,5)$ & \\
\hline \multicolumn{4}{|l|}{ Causa externa, $n(\%)$} \\
\hline FAB & $4(3,3)$ & $2(2,8)$ & \multirow{4}{*}{$0,843^{\mathrm{QM}}$} \\
\hline FAF & $8(6,7)$ & $8(11,1)$ & \\
\hline Acidente de Transporte & $91(75,8)$ & $51(70,8)$ & \\
\hline Agressão & $9(7,5)$ & $5(6,9)$ & \\
\hline \multicolumn{4}{|l|}{ Queda } \\
\hline \multicolumn{4}{|c|}{ Atendimento pré-hospitalar, $n(\%)$} \\
\hline Sim & $117(97,5)$ & $68(94,4)$ & \multirow{2}{*}{$0,428^{\mathrm{F}}$} \\
\hline Não & $3(2,5)$ & $4(5,6)$ & \\
\hline
\end{tabular}

Legenda: $\mathrm{n}$ - frequência absoluta. \% - frequência relativa percentual. DP - Desvio Padrão. F Teste Exato de Fisher. QM Teste Qui-Quadrado de Pearson com simulações de Monte-Carlo. W Teste de Mann-Whitney.

Fonte: Autores.

No grupo TRISS-like com relação as regiões anatômicas (AIS), a face (50\% vs 38,9\%, p=0,135) e extremidades ou cintura pélvica $(44,2 \%$ vs $51,4 \%, p=0,332)$ foram as mais acometidas. No grupo TRISS a classificação do ECG teve média de 14,6 (DP=12,1), a FR uma média de 19,8(DP=5,9), e o RTS obteve média de 7,5 (DP=0,4). No TRISS-like a MRM obteve média de 3,3 (DP=1,4). O TRISS-like apresentou maior média de ISS do que o TRISS (14,5 vs 10,3, p<0,001). A média nos níveis pressóricos sistólicos não houve diferença significativa quando comparado o TRISS com o TRISS-like (p =0,124), nos escores da pressão acima de $89 \mathrm{mmHg}(90,8 \%$ vs $100 \%$, p=0,027) com diferença significativa (Tabela 4). 
Tabela 4 - Dados comparativos entre os pacientes vítimas de TCE do grupo TRISS-like e do grupo TRISS admitidos no setor crítico do HUSE, no período de Agosto de 2018 a Julho de 2019, segundo características das regiões corpóreas acometidas e fisiológicas. Aracaju/SE, Brasil. (N=192).

\begin{tabular}{|c|c|c|c|}
\hline & \multicolumn{2}{|c|}{ Tipo } & \multirow[b]{3}{*}{ p-valor } \\
\hline & \multirow[t]{2}{*}{$\begin{array}{c}\text { TRISS-like } \\
(\mathbf{n}=120)\end{array}$} & \multirow[t]{2}{*}{$\begin{array}{l}\text { TRISS } \\
(n=72)\end{array}$} & \\
\hline & & & \\
\hline \multicolumn{4}{|l|}{ AIS } \\
\hline Face & $60(50,0)$ & $28(38,9)$ & $0,135^{Q}$ \\
\hline Tórax & $30(25,0)$ & $13(18,1)$ & $0,264 \mathrm{Q}$ \\
\hline Abdomen ou Conteúdo Pélvico & $11(9,2)$ & $7(9,7)$ & $1,000^{\mathrm{F}}$ \\
\hline Extremidades ou Cintura Pélvica & $53(44,2)$ & $37(51,4)$ & $0,332^{Q}$ \\
\hline Superfície Externa & $6(5,0)$ & $2(2,8)$ & $0,712^{\mathrm{F}}$ \\
\hline ECG, média $(D P)$ & - & $14,6(12,1)$ & \\
\hline \multicolumn{4}{|l|}{ COD. ECG, $n(\%)$} \\
\hline $9-12$ & - & $25(34,7)$ & \\
\hline $13-15$ & - & $47(65,3)$ & \\
\hline MRM, média $(D P)$ & $3,3(1,4)$ & - & \\
\hline FR, média $(D P)$ & - & $19,8(5,9)$ & \\
\hline \multicolumn{4}{|l|}{ COD. FR, $n(\%)$} \\
\hline $10-29 \mathrm{inc} / \mathrm{min}$ & - & $67(93,1)$ & \\
\hline PAS, média $(D P)$ & $120,0(28,4)$ & $126,7(23,1)$ & $0,124^{\mathrm{w}}$ \\
\hline \multicolumn{4}{|l|}{ COD.PAS, $n(\%)$} \\
\hline $50-75 \mathrm{mmHg}$ & $7(5,8)$ & $0(0)$ & \\
\hline $\begin{array}{l}76-89 \mathrm{mmHg} \\
>89 \mathrm{mmHg}\end{array}$ & $\begin{array}{c}4(3,3) \\
109(90,8)\end{array}$ & $\begin{array}{c}0(0) \\
72(100)\end{array}$ & $0,027 \mathrm{QM}$ \\
\hline ISS, média (DP) & $15,1(9,4)$ & $10,5(9,6)$ & $<0,001$ w \\
\hline RTS, média (DP) & - & $7,5(0,4)$ & \\
\hline
\end{tabular}

Legenda: $\mathrm{n}$ - frequência absoluta. \% - frequência relativa percentual. DP - Desvio Padrão. F Teste Exato de Fisher. Q Teste Qui-Quadrado de Pearson. QM Teste Qui-Quadrado de Pearson com simulações de Monte-Carlo. W Teste de Mann-Whitney.

Fonte: Autores.

Na Tabela 5 considerou-se que os pacientes de TCE avaliados pelo método TRISS-like, tiveram uma maior prevalência de procedimentos cirúrgicos realizados em menos de 24 horas com 54 (45,4\%), e em procedimentos realizados na cabeça 31 (57,4\%). Observou-se uma prevalência significativa para o grupo TRISS-like quanto a sequelas (22,2\% vs 6,9\%, $\mathrm{p}=0,017)$, óbitos $(45 \%$ vs $16,7 \%, \mathrm{p}<0,001)$ e internação em UTI $(52,5 \%$ vs 34,7\%, p=0,017). No método TRISS houve uma diferença significativa em relação a retirada cirúrgica de órgãos (0\% vs 40\%, p=0,022). 
Tabela 5 - Dados comparativos entre os pacientes vítimas de TCE do grupo TRISS e do grupo TRISS-like admitidos no setor crítico do HUSE, no período de Agosto de 2018 a Julho de 2019, segundo características do evento traumático, consequências e desfecho. Aracaju/SE, Brasil. (N=192).

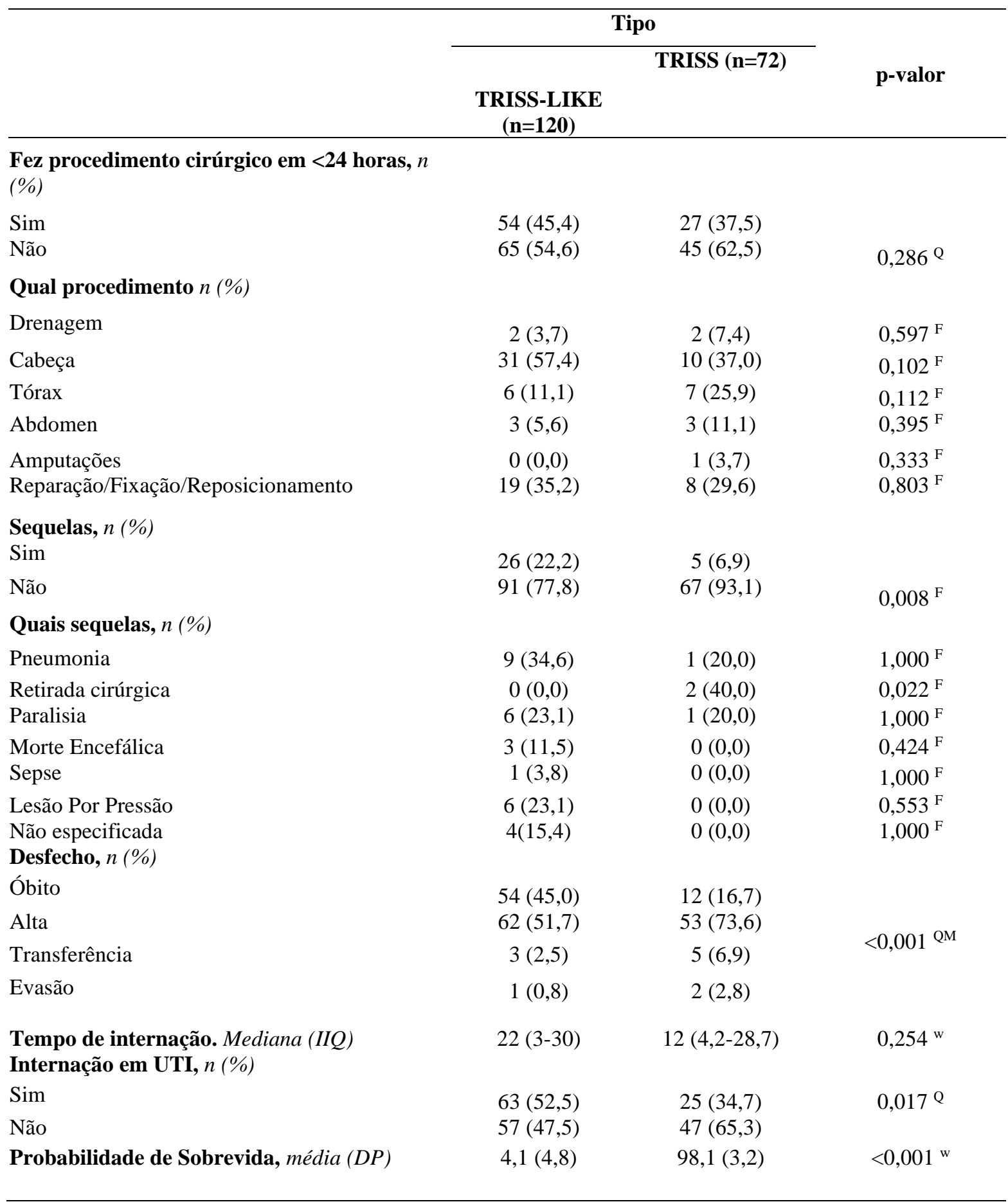

Legenda: $\mathrm{n}$ - frequência absoluta. \% - frequência relativa percentual. DP - Desvio Padrão. F Teste Exato de Fisher. Q Teste Qui-Quadrado de Pearson. QM Teste Qui-Quadrado de Pearson com simulações de Monte-Carlo. W Teste de Mann-Whitney.

Fonte: Autores.

$\mathrm{Na}$ Tabela 6 são apresentados os resultados do modelo de regressão logística para TRISS-like. A princípio, foram incluídas no modelo todas as variáveis significativas a $20 \%$, que não apresentavam nenhum problema com separação parcial ou total cuja quantidade de observações sem dado não ultrapassasse os $10 \%$ no conjunto de variáveis. Após esta etapa foram calculadas as razões de chances brutas, onde o desfecho óbito, internado em UTI, ter sequelas, maior ISS aumentam as chances 
de o paciente ser TRISS-like isoladamente. Após esta etapa, foi construído um modelo múltiplo, onde utilizou-se o método de Backward selection para escolha das variáveis ao nível de 5\% conjuntamente. O modelo final é composto de desfecho e sequelas, onde pacientes que foram a óbito e tiveram sequelas simultaneamente aumentam as chances de ser TRISS-like.

Tabela 6 - Dados de regressão logística dos pacientes vítimas de TCE do grupo TRISS-like admitidos no setor crítico do HUSE, no período de Agosto de 2018 a Julho de 2019, segundo faixa etária, desfecho, internação em UTI e sequelas. Aracaju/SE, Brasil. ( $\mathrm{N}=120)$.

\begin{tabular}{|c|c|c|c|}
\hline & RC (IC95\%) & RCa (IC95\%) & p-valor \\
\hline \multicolumn{4}{|l|}{ Faixa etária } \\
\hline $18-29$ & $1,21(0,39-3,78)$ & & \\
\hline $30-44$ & $2,25(0,70-7,27)$ & & \\
\hline $45-59$ & $2,18(0,61-7,75)$ & & \\
\hline$>60$ & 1 & & \\
\hline \multicolumn{4}{|l|}{ Desfecho } \\
\hline Óbito & $4,04(1,95-8,36)$ & $3,87(1,86-8,09)$ & $<0,001$ \\
\hline Transferência & $0,54(0,12-2,36)$ & $0,63(0,14-2,78)$ & 0,541 \\
\hline Evasão & $0,45(0,04-5,10)$ & $0,52(0,05-5,97)$ & 0,603 \\
\hline Alta & 1 & 1 & \\
\hline \multicolumn{4}{|c|}{ INTERNAÇÃO EM UTI } \\
\hline Sim & $1,98(1,08-3,63)$ & & \\
\hline Não & 1 & 1 & \\
\hline \multicolumn{4}{|l|}{ SEQUELAS } \\
\hline Sim & $3,83(1,40-10,45)$ & $3,30(1,17-9,28)$ & 0,024 \\
\hline Não & 1 & 1 & \\
\hline
\end{tabular}

Legenda: RC - Razão de Chances. RCa - Razão de Chances ajustadas. IC95\% - Intervalo com 95\% de confiança. Fonte: Autores.

\section{Discussão}

O presente estudo demonstrou que o TCE é mais prevalente na população masculina (87,5\%), dos quais $39,1 \%$ estavam numa faixa etária entre 18 e 29 anos, resultados semelhantes ao de Pereira (2006), que também teve como estudo a população de Sergipe, obtendo resultados na faixa etária entre 20 e 29 anos (45\%), com uma prevalência de $73 \%$ do sexo masculino. Os resultados do estudo também corroboram com o encontrado em outra pesquisa, no Ceará, obtendo resultados que mostraram $82 \%$ das vítimas pertencem ao sexo masculino, 22\% tem entre 21 e 30 anos de idade (Silva et al., 2017). No território brasileiro, Fernandes e Silva (2013), encontrou também um predomínio da população masculina (81.5\%), com uma distribuição de idade entre 14 e 34 anos (53\%). A forma como está distribuição se acontece está provavelmente relacionada às características sociais, econômicas e culturais da população, onde o jovem do sexo masculino se expõe com uma frequência muito maior a situações de risco, como a direção imprudente e inexperiente de automóveis, confrontos físicos, além da associação destes ao álcool, que, por prejudicar a atenção e interferir na percepção de perigo, favorece a exposição a situações de risco.

Como foi afirmado Hyder et al. (2007), a principal causa mundial do TCE é o acidente de trânsito, com o resultado achado no presente estudo seguindo este padrão, com $73 \%$ dos casos derivados desta etiologia. Estudo anterior no estado de Sergipe, encontrou um valor de 64,8\% de acidentes de trânsito, mostrando não somente a manutenção desta etiologia, como também o aumento de sua prevalência no estado ao longo dos anos (Vaez et al., 2015). Outros estudos recentes em estados do 
Nordeste como Santos et al. (2016), encontraram uma prevalência de 85\% no estado do Piauí, já de Moura et al. (2011), encontrou 66,28\% em Petrolina, no estado de Pernambuco. Outras das etiologias mais encontradas foram queda (própria ou de grande altura) e a agressão física, cuja prevalência como segunda maior causa varia de acordo com o local onde o estudo foi realizado (Viégas et al., 2013). O mecanismo de trauma em sua maioria foi por contuso (89,1\%), muito devido a biomecânica envolvida nesses acidentes. Algumas medidas que poderiam diminuir o envolvimento desta parcela da população nos casos de TCE já foram implementadas, a exemplo da Lei n 11.705, de 19 de junho de 2008, também chamada de "Lei Seca". Outras medidas, seriam por meio da educação no trânsito, além de maiores medidas de fiscalização nas rodovias, como reforçar o uso de capacetes em pilotos de motocicletas, e cintos de segurança em motoristas de uma forma geral.

No presente estudo, foi visto que 96,4\% dos pacientes receberam atendimento pré-hospitalar, logo, infere-se o potencial para bom prognostico destes pacientes. O achado corrobora com o de outros, como Praça et al. (2017), onde foi mostrado dos pacientes teve atendimento pré-hospitalar, incluindo medidas de oxigenoterapia adequadas ao seu quadro clínico. A precoce e adequada realização do atendimento pré-hospitalar é um ponto positivo para evitar danos secundários, devendo sempre tentar otimizar sua aplicação. Segundo Moita e Ferreira (2018), o atendimento pré-hospitalar é referenciado como um dos mais importantes redutores de mortalidade na população ativa.

É importante observar que apesar do percentual de pacientes que tiveram necessidade de internação em unidade de tratamento intensivo (UTI) tenha sido menor que os dos indivíduos onde não houve essa necessidade (45,8 e 54,2\% respectivamente), os resultados obtidos mostram valores muito próximos, não sendo exatamente tranquilizadores. Quando comparados a estudos de outros estados, como Silva et al. (2017) no Ceará, cujo resultados mostraram que apenas 5\% dos casos pesquisados foram internados na UTI, vemos que pode haver uma falha na questão de admissão neste setor do hospital, sendo possível a ocorrência de internações desnecessárias em UTI, as quais devem estar reservadas para casos que obedeçam aos devidos critérios para admissão. Pode se ainda estar ligado ao número reduzido desse tipo de leito nem outros estados. Fazse então necessário uma melhor implementação da triagem a qual esses pacientes são submetidos, de modo que apenas aqueles que realmente precisam de leito em UTI sejam admitidos neste setor, minimizando gastos, e evitando lotação dessas unidades.

Quanto ao desfecho dos pacientes, houve uma prevalência de 59,9\% de altas hospitalares no estudo, comparado à 34,4\% que, infelizmente, foram à óbito. No estudo de Silva et al. (2017), realizado num hospital de referência em Fortaleza, os resultados encontrados foram mais discrepantes: $84 \%$ receberam alta, enquanto $16 \%$ dos pacientes foram a óbito, o estudo de Moura et al. (2011), por sua vez, aponta um resultado semelhante em Petrolina, com 88,12\% dos casos recebendo alta e um número ainda menor de óbitos de 7,92\%. Foi observado ainda uma média de 11 dias de internação, valor quase duas vezes maior com uma média de 5,99 dias (Moura et al., 2011). É possível que o maior número de óbitos encontrados em nossa casuística tenha sido ocasionado devido a própria natureza das lesões encontradas nos pacientes, as quais poderiam ter uma gravidade maior, além disso, comorbidades associadas a lesão do TCE podem também ter influenciado no desfecho destes pacientes, em sua maioria pneumonia (32,3\%). Para uma melhor assistência desses pacientes, sugiro uma descrição minuciosa de comorbidades, a exemplo do alcoolismo, além de parâmetros clínicos, laboratoriais e com o uso exames complementares de imagem como ressonância magnética, que seriam de fundamental importância para a identificação de marcadores prognósticos. Utilizar métodos diagnósticos adequados é fundamental para tomar a decisão sobre o tratamento do TCE, além de reduzir os custos.

Com relação ao tratamento e evolução do trauma, foi mostrado que na maior parte dos pacientes $(57,6 \%)$, foi optado por tratamento conservador, enquanto menos da metade dos pacientes incluídos na pesquisa (42,4\%) foram submetidos à tratamento cirúrgico antes das primeiras 24h. Tal achado corrobora com o resultado do estudo de Maia et al. (2013), onde o tratamento mais realizado nas vítimas de TCE foi o conservador $(77,18 \%)$. O achado pode decorrer devido à uma maior frequência de traumas leves e moderados. Este tipo de conduta é preferível devido ao grande ônus à saúde pública que o 
tratamento cirúrgico acarreta, uma vez que necessita de cuidados especializados, a recuperação do paciente é mais lenta, além do aumento da chance de o paciente desenvolver infecções, devendo reservar essa conduta para situações devidamente necessárias.

Quanto ao prognóstico dos pacientes avaliados pelo método TRISS-like, observou-se uma maior prevalência em procedimentos cirúrgicos realizados em menos de 24 horas $(45,4 \%)$, e em procedimentos realizados na cabeça $(57,4 \%)$, foi vista ainda uma prevalência significativa para o grupo TRISS-like quanto a sequelas ( $\mathrm{p}=0,017)$, óbitos $(\mathrm{p}<0,001)$ e internação em UTI. Já no método TRISS, houve uma diferença significativa em relação a retirada cirúrgica ( $\mathrm{p}=0,022$ ). De acordo com Sarani e Martin (2016), o TRISS pode predizer com precisão a probabilidade de sobrevida em pacientes vítimas de trauma contuso, corroborando com a casuística do presente estudo. O tipo e a gravidade das lesões, aspectos levados em consideração pelos métodos TRISS e TRISS-like, tendo sido reconhecidos como os fatores mais importantes na determinação prognóstica. Pode-se então dizer que o prognóstico é diretamente influenciado por vários fatores, tais como idade, condição de saúde préinjúria, tempo de decorrido entre o evento e a assistência definitiva, qualidade da assistência e complicações, devido a forma em que eles são implementados no cálculo desses métodos. A determinação da severidade do trauma é uma ferramenta essencial na condução do tratamento e para estabelecer os recursos necessários para prestar um melhor atendimento, logo, o uso de rotina dos métodos TRISS e TRISS-like seria um grande aliado na melhor condução nos casos de TCE.

\section{Conclusão}

Os achados do presente estudo reforçam que o perfil geral das vítimas foi predominantemente do sexo masculino, a adultos jovens na faixa etária entre 18 e 29 anos. Quanto a etiologia, o TCE teve como principal causa o acidente de transporte, de mecanismo contuso, atingindo em sua maioria as extremidades, cintura pélvica e região de face. Esta distribuição acontece provavelmente devido às características sociais, econômicas e culturais da população, onde o jovem do sexo masculino se expõe com uma frequência muito maior a situações de risco, como a direção imprudente e inexperiente de automóveis, confrontos físicos, além da associação destes ao álcool, que, por prejudicar a atenção e interferir na percepção de perigo, favorece a exposição a situações de risco.

A maioria dos pacientes receberam atendimento pré-hospitalar, tiveram tratamento conservador, além de não haver a necessidade de ir para UTI e, em sua maior parte, tiveram alta hospitalar sem sequelas. Entretanto foi evidenciada uma taxa de óbito elevada, o que denota perdas de anos de vida produtiva levando a consequências psicológicas e econômicas.

Os métodos TRISS e TRISS-like foram scores adequados na determinação prognóstica, sendo eficazes na determinação do desfecho dos pacientes estudados na presente casuística. No entanto, sugere-se futuros estudos com a utilização de outros índices como o NISS e o NTRISS-like, que complementam as limitações do TRISS, ainda que o método TRISS seja considerado padrão ouro.

\section{Referências}

Blackwell, D. L., Lucas, J. W., \& Clarke, T. C. (2014). Summary health statistics for US adults: national health interview survey, 2012. Vital and health statistics. Series 10, Data from the National Health Survey, (260), 1.

Champion, H. R., Copes, W. S., Sacco, W. J., Lawnick, M. M., Keast, S. L., \& Frey, C. F. (1990). The Major Trauma Outcome Study: establishing national norms for trauma care. Journal of Trauma and Acute Care Surgery, 30(11), 1356-1365.

Domingues, C. D. A. (2013). Trauma and injury severity score: análise de novos ajustes no índice (Doctoral dissertation, Universidade de São Paulo).

Estumano, G. R. W., Almeida, J. P. D. O., Feitosa Neto, P. A., \& Fontelles, M. J. P. (2015). Índices de trauma como método prognóstico em pacientes vítimas de trauma abdominal atendidos no Hospital de referência de Urgência e Emergência no estado do Pará. Rev. para. med., 29(3).

Fernandes, R. N. R., \& Silva, M. (2013). Epidemiology of traumatic brain injury in Brazil. Arquivos Brasileiros de Neurocirurgia: Brazilian Neurosurgery, 32(03), 136-142. 
Hyder, A. A., Wunderlich, C. A., Puvanachandra, P., Gururaj, G., \& Kobusingye, O. C. (2007). The impact of traumatic brain injuries: a global perspective. NeuroRehabilitation, 22(5), 341-353.

Maia, B. G., de Paula, F. R. P., Cotta, G. D., Cota, M. D. A. L., Públio, P. G., de Oliveira, H., \& de Oliveira, T. A. (2013). Perfil clínico-epidemiológico das ocorrências de traumatismo cranioencefálico. Revista Neurociências, $21(1), 43-52$.

Moita, C. E., \& Ferreira, R. M. (2018). O Papel Do Enfermeiro No Atendimento Pré-Hospitalar Do Paciente Com Trauma Crânio Encefálico. Revista De Trabalhos Acadêmicos-Universo Salvador, 1(6).

Moura, J. C., Rangel, B. L. R., Creôncio, S. C. E., \& Pernambuco, J. R. B. (2011). Perfil clínico-epidemiológico de traumatismo cranioencefálico do Hospital de Urgências e Traumas no município de Petrolina, estado de Pernambuco. Arquivos Brasileiros de Neurocirurgia: Brazilian Neurosurgery, 30(03), 99-104.

Parreira, J. G., Kanamori, L. R., Valinoto, G. C., \& Perlingeiro, J. A. (2014). Giannini SSC, Assef JC. Análise comparativa dos fatores preditivos de morte em vítimas de trauma fechado com fraturas pélvicas. Rev Col Bras Cir, 41(4), 285-91.

Pereira, C. U., Duarte, G. C., \& Santos, E. A. S. (2006). Avaliação epidemiológica do traumatismo craniencefálico no interior do Estado de Sergipe.

Pocock, S. J. (1983). Clinical trials: a practical approach. Chicester: John Wiley\&Sons.

Praça, W. R., Matos, M. C. B., da Silva Magro, M. C., \& de Souza Hermann, P. R. (2017). Perfil epidemiológico e clínico de vítimas de trauma em um hospital do Distrito Federal. Revista Prevenção de Infecção e Saúde, 3(1), 1-7.

Quevedo, M. J. S. (2009). Internações em UTI por trauma cranioencefálico (TCE) na cidade de Porto Alegre.

Raja, A., \& Zane, R. D. (2018). Initial management of trauma in adults.

Santos, A. M. R. D., Sousa, M. E. D. C., Lima, L. O., Ribeiro, N. D. S., Madeira, M. Z. D. A., \& Oliveira, A. D. D. S. (2016). Perfil epidemiológico do trauma cranioencefálico. Rev. enferm. UFPE on line, 3960-3968.

Sarani, B., \& Martin, N. (2016). Overview of inpatient management in the adult trauma patient. UpToDate. Dostopno januarja.

Silva, J. A. D., Souza, A. R. D., Feitoza, A. R., \& Cavalcante, T. D. M. C. (2017). Traumatismo cranioencefálico no município de Fortaleza. Enferm. foco (Brasília), 22-26.

Sousa, R. M. C. D. (2006). Comparação entre instrumentos de mensuração das consequências do trauma crânio-encefálico. Revista da Escola de Enfermagem da USP, 40(2), 203-213.

Vaez, A. C., de Vasconcelos, J. M., de Jesus, L. K. A., Pinheiro, F. G. D. M. S., de Paula, C. L. P., \& da Conceição Araújo, D. (2015). Perfil clínico epidemiológico das vítimas de trauma cranioencefálico no intra-hospitalar de um hospital público do estado de Sergipe. Caderno de Graduação-Ciências Biológicas e da Saúde-UNIT-SERGIPE, 3(1), 113-126.

Viégas, M. L. C., Pereira, E. L. R., Targino, A. A., Furtado, V. G., \& Rodrigues, D. B. (2013). Traumatismo cranioencefálico em um hospital de referência no estado do Pará, Brasil: prevalência das vítimas quanto a gênero, faixa etária, mecanismos de trauma, e óbito. Arquivos Brasileiros de Neurocirurgia: Brazilian Neurosurgery, 32(01), 15-18. 